\title{
An Extended Network Model with a Packages Diffusion Process
}

\author{
C.Pigorsch and S.Trimper \\ Martin-Luther-Universität Halle-Wittenberg, \\ Fachbereich Physik, 06099 Halle Germany
}

October 28, 2018

\begin{abstract}
The dynamics of a packages diffusion process within a selforganized network is analytically studied by means of an extended $f$-spin facilitated kinetic Ising model (Fredrickson-Andersen model) using a Fock-space representation for the master equation. To map the three component system (active, passive and packages cells) onto a lattice we apply two types of second quantized operators. The active cells correspond to mobile states whereas the passive cells correspond to immobile states of the Fredrickson-Andersen model. An inherent cooperativity is included assuming that the local dynamics and subsequently the local mobilities are restricted by the occupation of neighboring cells. Depending on a temperature-like parameter $h^{-1}$ (interconnectivity) the diffusive process of the packages (information) can be almost stopped, thus we get a well separation of the time regimes and a quasi-localization for the intermediate range at low temperatures.
\end{abstract}

\section{Introduction}

During the last years there is a huge effort to understand the kinetics of nonequilibrium phenomena. A wide range of discrete and continuous models for such processes is analytically and numerically studied. The problems of 
interest in this context concern the crystal growth, transport (traffic) models, diffusion processes and supercooled liquids [1 6].

Here, we will apply the kinetics of the Fredrickson-Andersen model (FAM) recently discussed in the framework of the glass transition and related phenomena [5 12]. But we show that this model may be used on other fields like stock trading, citation networks, company relations or internet communications as well 13 16. In general, we study the diffusion of information within a network system of active links and passive/active cells (or nodes). The switch between a passive and active cell is controlled by a temperaturelike parameter $h^{-1}$ which can be interpreted as the interconnectivity. At the maximum interconnectivity (at infinite temperature) the system possesses equal parts of active and passive cells (nodes) whereas at the minimum interconnectivity (at zero temperature) there are only passive cells (nodes). Furthermore, the alteration is also controlled by the nearest neighborhood. If enough adjacent active cells (nodes) exist (more than a fixed number $f$ ) the active cell (node) can become passive and vice versa. Thus, only a sufficiently active environment may determine and alter the state of a cell (node) like in a citation community where only accepted (active) people may decide about the worth of an opinion of a member in a related field. To this network formed by passive/active cells (nodes) and active links, consisting of two adjacent active cells (nodes), we add further particles which may be assigned to (information) packages. These particles can only diffuse along active links but are confined by passive cells (nodes). Therefore, we have a diffusion in a self-organized network where information may stick (and therefore be localized) at passive cells (nodes) or runs through a network of active links. This is the same situation like in the internet where passive routers cannot transfer any data but data on active router should be directed to the next active router (Of course, the difference is that this is a directed motion whereas we consider a non-directed motion here.).

Now we demonstrate how one may relate this network to the FAM originally dedicated to supercooled liquids. For such systems one assumes that the dynamics can be roughly regarded as independent of the microscopic details, thus the analysis is based on a mesoscopic formulation. Hence, one reduces the degrees of freedom to a smaller set of relevant observables following the original idea of Fredrickson and Andersen [7 9]. The supercooled liquid as the many-body system is treated on a virtual lattice with sufficiently small cells of size $l$ where the lattice should not have any significant influence on 
the underlying dynamics. However, in case of our network model we will immediately start on the mesoscopic scale (e.g. on the elementary scale of routers where the macroscopic scale is the total internet).

The cell structure enables us to attach to each cell $j$ a two-state observable $\sigma_{j}$ which characterizes its actual state. The realization is given by the local activity $m_{j}$ with $\sigma_{j}=-1$ (spin down) if $m_{j}<\bar{m}$ (passive state) and $\sigma_{j}=+1$ (spin up) if $m_{j}>\bar{m}$ (active state) where $\bar{m}$ is a fixed threshold of the system. The threshold is chosen in this way that all cells are passive at zero temperature. Active (passive) states may be also denoted as mobile (immobile) states. The number of cells in the active and the passive state, respectively, have not to be conserved. (Notice that in case of supercooled liquids the usual separation is done by two different density states although this is more connected to a Kawasaki exchange dynamics rather than to a Glauber flip dynamics [9]. This mapping implies different densities of such cells; $\sigma_{j}=-1$ corresponds to the more solid-like state and $\sigma_{j}=1$ corresponds to the more liquid-like state.) Thus, the real kinetics is more Glauber-like (non-conserved) dynamics thus we suppose that the basic dynamics is a flip process $\sigma_{j}=+1 \leftrightarrow \sigma_{j}=-1$. It is controlled by self-induced topological restrictions introduced below. This type of dynamics leads to a relaxation behavior resembled to that of a cooperative system. In particular, an elementary switch at a given cell is allowed only if the number of nearest neighbor mobile/active cells $\left(\sigma_{j}=+1\right)$ is equal to or larger than a certain restrictive number $f$ with $0 \leq f \leq z$ ( $z$ is the coordination number of the lattice). Hence, elementary flip processes combined with the restrictions may lead to the cooperative arrangements within the underlying network system.

As demonstrated such a mesoscopic approach is able to model supercooled liquids as well. The Fredrickson-Andersen model (or $f$-spin facilitated kinetic Ising model) [7,9] was analytically [5,6,17] and numerically 10 12, 18, studied in broad variety. The FAM can be classified as an Ising-like model of which kinetics is confined by restrictions of the ordering of nearest neighbors to a given lattice cell. These self-adapting environments especially influence the long-time behavior of the spin-relaxation [6, 11] particularly relevant for supercooled liquids.

Additionally, here we add particles to the system of which diffusive dynamics are coupled with the existence of the active states. Therefore, we require that active cells are necessary for the motion of packages because passive states block any packages diffusion. Or in other words data are fixed 
and static as long as they are assigned to passive cells, but information may diffuse through a network of active cells. In the present work we will incorporate this features into the Fock-space representation of the master equation in order to compute the packages concentration in a continuous mean-field-like approximation.

\section{Algebraic Representation and Master Equa- tion}

Firstly, we give a short survey about the Fock-space method (known as quantum Hamiltonian as well - more details can be found in e.g. [3]) A certain state in our lattice system can be characterized by a set of discrete numbers $\vec{n}=\left\{n_{i}\right\}$ (or resp. $\vec{v}=\left\{v_{i}\right\}$ ) where $n_{i}, v_{i} \in\{0,1\}$ denote the local state of a lattice cell $i$. Furthermore, the following convention is used: $n_{i}=1(0)$ is related to the active (passive) state, also denoted by $A(B)$. The state $v_{i}=1$ (0) corresponds to a cell $i$ occupied (non-occupied) by a package. We start from the one-step master equation formulated in the following form

$$
\partial_{t} P(\vec{n}, \vec{v}, t)=L^{\prime} P(\vec{n}, \vec{v}, t)
$$

where $P(\vec{n}, \vec{v}, t)$ is the probability for a certain configuration $\{\vec{n}, \vec{v}\}$. The linear operator $L^{\prime}$ specified by the dynamics of the system describes the time evolution. Then, following [19,20], the probabilities $P(\vec{n}, \vec{v}, t)$ can be related to the Fock-space state vector $|F(t)\rangle$ as a weight using the decomposition into the base vectors $|\vec{n}\rangle \otimes|\vec{v}\rangle$ of a orthonormal vector space,

$$
|F(t)\rangle=\sum_{\vec{n}, \vec{v}} P(\vec{n}, \vec{v}, t)|\vec{n}\rangle \otimes|\vec{v}\rangle .
$$

This equation enables the quantum formulation for the master equation resulting in an equivalent manner

$$
\partial_{t}|F(t)\rangle=\hat{L}|F(t)\rangle
$$

where $L^{\prime}$ is assigned to the operator $\hat{L}$ in the Fock-space representation. The procedure had been originally derived for Bose-like system [19, 20] and was later applied also to Fermi-like systems [4,21,22]. Recently, we have proposed 
a further extension applying Para-Fermi statistics for different restricted occupation numbers 6,23 . The average of a physical quantity $G(\vec{n}, \vec{v})$ is given by the trace over $G$ :

$$
\langle\hat{G}(t)\rangle=\sum_{\vec{n}, \vec{v}} P(\vec{n}, \vec{v}, t) G(\vec{n}, \vec{v})=\langle\vec{r}|\hat{G}| F(t)\rangle
$$

where $\langle\vec{r}|$ is the reference state related to the base vectors by

$$
\langle\vec{r}|=\sum_{\vec{n}, \vec{v}}\langle\vec{n}| \otimes\langle\vec{v}|=\bigotimes\left(\begin{array}{l}
1 \\
1
\end{array}\right) .
$$

The reference state is completely determined by the base $\{|\vec{n}\rangle,|\vec{v}\rangle\}$ of the Fock space and not assigned to the special model or to the evolution operator $\hat{L}$. The conservation of the total probability is manifested by $\langle\vec{r}| \hat{L}=0$. Therefore, the equation of motion is given by

$$
\partial_{t}\langle\hat{G}(t)\rangle=\langle\vec{r}|\hat{G} \hat{L}| F(t)\rangle=\left\langle\vec{r}\left|[\hat{G}, \hat{L}]_{-}\right| F(t)\right\rangle .
$$

Notice that the dynamical equation depends on both the algebraic properties of the underlying operators and the mathematical structure of $\hat{L}$. Thus, we introduce the second quantized lowering $a_{i}\left(v_{i}\right)$ and raising $a_{i}^{\dagger}\left(v_{i}^{\dagger}\right)$ operators forming the evolution operator $\hat{L}$ to create the base states $|\vec{n}\rangle(|\vec{v}\rangle)$ from a vacuum state $|0\rangle$. Both types of (independent from each other and hence commuting) operators fulfil the relationship (for Paulions)

$$
a_{i} a_{j}^{\dagger}+a_{j}^{\dagger} a_{i}=\delta_{i, j}+2 a_{j}^{\dagger} a_{i}\left(1-\delta_{i, j}\right) \text {. }
$$

As mentioned above, the inherent properties of the FAM is the restriction of the flip dynamics at cell $i, \sigma_{i} \leftrightarrow-\sigma_{i}$,

$$
\frac{1}{2} \sum_{j(i)}\left\langle n_{j}\left|\left(1+\sigma_{j}\right)\right| n_{j}\right\rangle=\sum_{j(i)}\left\langle n_{j}\left|\hat{A}_{j}\right| n_{j}\right\rangle \geq f
$$

where $j(i)$ means a sum over all adjacent cells of $i$ and $f$ is the restriction number. The number operators $\hat{A}_{j}$ and $\hat{V}_{j}$ denote $a_{j}^{\dagger} a_{j}$ and $v_{j}^{\dagger} v_{j}$ as usual. Concerning the motion of information packages we postulate a diffusive motion of the particles coupled to the existence of active cells at both related 
sites. This exchange process due to Kawasaki enhances the mobility of packages in active neighborhoods whereas it slows down inside a passive cluster. Summarizing we obtain the evolution operator taking the form

$$
\begin{aligned}
L= & L_{F}+L_{E} \\
L_{F}= & +\sum_{i} \lambda_{B A}\left(1-a_{i}\right) a_{i}^{\dagger} \sum_{<m_{1} \cdots m_{f}, i>} \hat{A}_{m_{1}} \cdots \hat{A}_{m_{f}} \\
& +\sum_{i} \lambda_{A B}\left(1-a_{i}^{\dagger}\right) a_{i} \sum_{<m_{1} \cdots m_{f}, i>} \hat{A}_{m_{1}} \cdots \hat{A}_{m_{f}} \\
L_{E}= & +\sum_{<r s>} D_{0}\left[v_{r}^{\dagger} v_{s}-\left(1-\hat{V}_{r}\right) \hat{V}_{s}\right] \hat{A}_{r} \hat{A}_{s}+\text { symmetric term }
\end{aligned}
$$

with the quantities $\lambda_{A B}=\tilde{\lambda} \exp [h], \lambda_{B A}=\tilde{\lambda} \exp [-h]$ and $D_{0}$ are the kinetic coefficients of the diffusion process. They are appropriate thermodynamic weighted to fulfil the detailed balance condition. The parameter $h$ corresponds to the inverse temperature of a heat bath and describes the interconnectivity between the cells. The higher $h$ is set the lower is the interconnectivity of the network. The first term of $L_{F}$ reflects the flip process $\sigma_{i}=-1 \rightarrow \sigma_{i}=+1$ whereas the second term represents $\sigma_{i}=+1 \rightarrow \sigma_{i}=-1$. The second part, $L_{E}$, expresses the exchange process $V_{i}+V_{j} \leftrightarrow V_{j}+V_{i}$ related to the existence of an active link between adjacent cells. The term in Eq.(9)

$$
\sum_{<m_{1} \cdots m_{f}, i>} \hat{A}_{m_{1}} \cdots \hat{A}_{m_{f}}
$$

represents the kinetic restriction which are mentioned above. The abbreviation $<m_{1} \cdots m_{f}, i>$ denotes the sets of all the $f$ lattice cells neighbored to the cell $i$. The operator $\hat{A}_{m}$ yields a non-zero result only if the cell $m$ is an active one, so that the expression (10) differs from a zero value if it is applied to a cell of interest surrounded by at least $f$ active cells.

Using Eq.(14) the temporal evolutions of the two relevant observables $\left\langle\hat{A}_{k}\right\rangle$ and $\left\langle\hat{V}_{k}\right\rangle$ result in

$$
\begin{aligned}
\partial_{t}\left\langle\hat{A}_{k}\right\rangle & =\lambda_{B A} \sum_{<m_{1} \cdots m_{f}, k>}\left\langle\hat{B}_{k} \hat{A}_{m_{1}} \cdots \hat{A}_{m_{f}}\right\rangle-\lambda_{A B} \sum_{<m_{1} \cdots m_{f}, k>}\left\langle\hat{A}_{k} \hat{A}_{m_{1}} \cdots \hat{A}_{m_{f}}\right\rangle \\
\partial_{t}\left\langle\hat{V}_{k}\right\rangle & =2 D\left\langle\nabla_{k}\left(\hat{A}_{k}^{2} \nabla_{k} \hat{V}_{k}\right)\right\rangle
\end{aligned}
$$

where we exploit the discrete form of the Laplacian

$$
\triangle_{k} O_{k}=\frac{1}{l^{2}} \sum_{r(k)}\left(O_{r}-O_{k}\right) .
$$


The diffusion coefficient is modified, $D=D_{0} l^{2}$, where $l$ is the length of the lattice cell. The current for the diffusive motion of the packages is given by

$$
j=-2 D \hat{A}_{k}^{2} \nabla_{k} \hat{V}_{k}
$$

It is intuitively obvious that the effective diffusion coefficient should be dependent on the squared concentration of the active cells, and therefore on the number of active links.

\section{Mean-field solution}

Because the formula for the vacancies concerns the same lattice index we may neglect it. Within the next step we apply a mean-field approximation and decouple all average values. Then, we obtain for the evolution equation of the packages

$$
\partial_{t}\langle V(t)\rangle=2 D \nabla\left[\langle A(t)\rangle^{2} \nabla\langle V(t)\rangle\right]
$$

whereas the equation of motion for the mobile/active state yields

$$
\partial_{t}\langle A(t)\rangle=\lambda_{B A} \zeta\langle 1-A(t)\rangle\langle A(t)\rangle^{f}-\lambda_{A B} \zeta\langle A(t)\rangle^{f+1} .
$$

Its temporal solution is easily given by

$$
\langle A(t)\rangle=\bar{A}+[A(0)-\bar{A}] \exp \left(-\frac{t}{\tau_{1}}\right)
$$

with the initial value $A(0)$ and the steady state solution

$$
\bar{A}=\frac{\lambda_{B A}}{\lambda_{A B}+\lambda_{B A}}=\frac{1}{\exp (2 h)+1} .
$$

As the inverse relaxation time of the flip process we find

$$
\tau_{1}^{-1}=\left(\lambda_{B A}+\lambda_{A B}\right) \zeta \bar{A}^{f}
$$

with $\zeta=z \cdot \ldots \cdot(f+1)$. Notice that the steady state solution in mean-field approximation is equal to the solution for the paramagnetic lattice gas and

independent of $f$. In contrast, the relaxation time depends on $\bar{A}^{f}$. Because 
the dynamics of the packages is globally conserved the steady solution is fixed for all time by the initial distribution, i.e.

$$
\bar{V}=\frac{\int V(x, 0) d x}{\int d x} .
$$

Testing the stability we analyze the Eqs.(14) and (15) in a linear manner. Assuming $\delta A$ and $\delta V$ are small fluctuations around the steady-state values we obtain by means of $\langle A(q, t)\rangle=\bar{A}+\delta A(\vec{q}, t)$ and $\langle V(\vec{q}, t)\rangle=\bar{V}+\delta V(\vec{q}, t)$ $(\vec{q}$ is the Fourier transformation of the local variable $\vec{x})$

$$
\partial_{t}\left(\begin{array}{l}
\delta A(\vec{q}, t) \\
\delta V(\vec{q}, t)
\end{array}\right)=-\left(\begin{array}{cc}
\tau_{1}^{-1} & 0 \\
0 & 2 D \bar{A}^{2} q^{2}
\end{array}\right)\left(\begin{array}{l}
\delta A(\vec{q}, t) \\
\delta V(\vec{q}, t)
\end{array}\right)
$$

The divergence of the second relaxation time $\tau_{2}^{-1}=2 D \bar{A}^{2} q^{2}$ at the wave number $\vec{q}=\overrightarrow{0}$ reflects the global conservation of the information in our network. Due to the quadratic dependence on $\bar{A}$ the relaxation time $\tau_{2}$ rapidly increases if the active cells become passive. Obviously, the steady state reveals as stable against pertubations indicated by the negative sign. To gain more insight into the diffusion process of the information associated with packages we consider its evolution equation (14) applying the mean-field solution for the active state (16). This leads to a spatial independent, but temporal dependent effective diffusion coefficient with

$$
\partial_{t}\langle V(t)\rangle=2 D\left[\bar{A}+(A(0)-\bar{A}) \exp \left(-\frac{t}{\tau_{1}}\right)\right]^{2} \nabla^{2}\langle V(t)\rangle .
$$

A measure for the fluctuations of the packages concentration is given by

$$
\begin{aligned}
F(t) & =\int_{0}^{t}\left\langle A\left(t^{\prime}\right)\right\rangle^{2} d t^{\prime} \\
& =\bar{A}^{2} t+2 \tau_{1} \bar{A}(A(0)-\bar{A})\left(1-e^{-\frac{t}{\tau_{1}}}\right)+\frac{\tau_{1}}{2}(A(0)-\bar{A})^{2}\left(1-e^{-\frac{2 t}{\tau_{1}}}\right) .
\end{aligned}
$$

Studying the asymptotic limits of $F(t)$ we recognize different temporal regimes. Whereas for small times the fluctuations are dominated by the initial value of the active cells $F(t) \sim A^{2}(0) t$ the fluctuations are approximated by $F(t) \sim$ $\bar{A}^{2} t$ for long times. Although the fluctuations go to infinity in the long-time limit (in this mean-field theory) there is a (quasi-)localization in an intermediate temporal range especially for small interconnectivity (equivalent to 
low temperature). Only a small part of all cells is active $(\bar{A} \ll(A(0)-\bar{A}))$ inhibiting the diffusion process. The exponential functions of the second and the third term in Eq.(22) are negligible so that the fluctuations $F(t)$ remains almost constant. To see if there is a quasi-localization one must compare the relaxation time $\tau$ in Eq.(18) and the time scale $t_{L}$ where the first term is equal the second and the third term in Eq.(22). In this connection, we assume that the exponential functions may be neglected. The time $t_{L}$ associated with this point is (supposing $A(0)>\bar{A}$ )

$$
t_{L}=\frac{\tau_{1}}{2}\left[\left(\frac{A(0)}{\bar{A}}\right)^{2}-1\right]
$$

Obviously, a localization $\left(t_{L} \gg \tau_{1}\right)$ becomes only possible if the concentration $\bar{A}$ is small enough (sufficient low interconnectivity/temperature) for the time interval $\tau_{1}<t<t_{L}$. A rough estimation yields the ratio

$$
\frac{t_{L}}{\tau_{1}} \sim \exp (4 h)
$$

Further, we may use $F(t)$ to transform the partial temporal derivative of the evolution equation,

$$
\frac{\partial}{\partial t}=\frac{d F(t)}{d t} \frac{\partial}{\partial F(t)}=\langle A(t)\rangle^{2} \frac{\partial}{\partial F(t)},
$$

and hence to obtain the ordinary diffusion equation

$$
\frac{\partial\langle V(t)\rangle}{\partial F(t)}=2 D \nabla^{2}\langle V(t)\rangle
$$

applying the transformed Eq.(14)

$$
\frac{\partial\langle V(t)\rangle}{\partial t}=\langle A(t)\rangle^{2} \frac{\partial\langle V(t)\rangle}{\partial F(t)}=2 D\langle A(t)\rangle^{2} \nabla^{2}\langle V(t)\rangle .
$$

If we now start with a $\delta$-distributed information (packages) density, i.e. we drop down the information at one point and observe how the information will be distributed in the time,

$$
V(\vec{x}, 0)=\bar{V} \delta(\vec{x})
$$


we get the result of the Eq.(14)

$$
V(\vec{x}, t)=\frac{1}{(4 \pi D F(t))^{\frac{d}{2}}} \exp \left(\frac{\vec{x}^{2}}{4 \pi D F(t)}\right) .
$$

Hence, the behavior of $F(t)$ directly influences the diffusion of the information. If $F(t)$ remains almost constant in a time regime the diffusion process and therefore the distribution of the information stops, and data packages are localized (compare Fig.11).

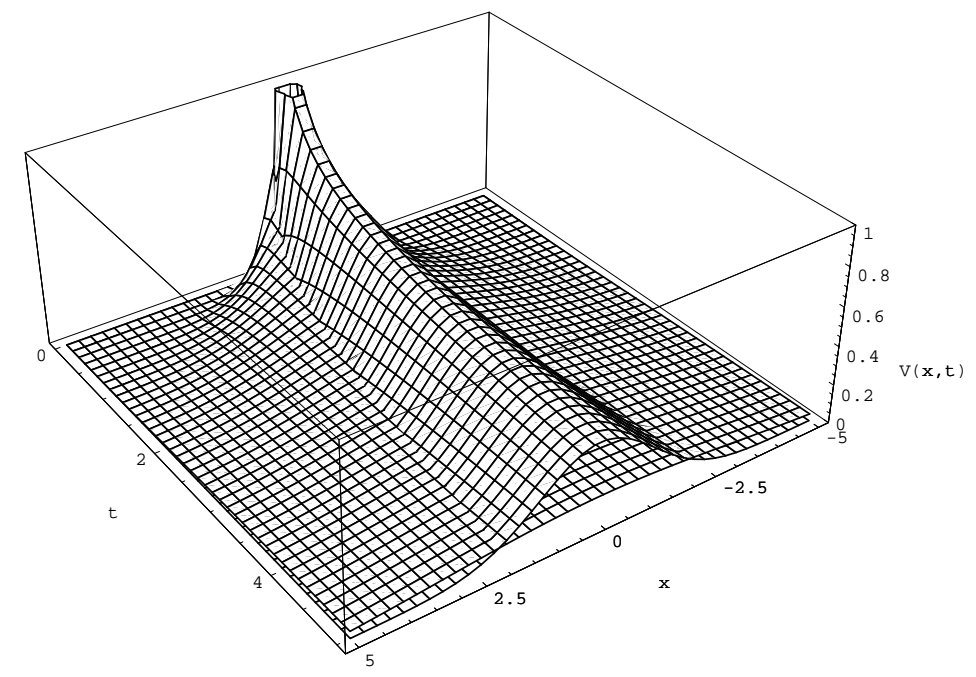

Figure 1: The diffusion of the packages concentration in the restricted network model (upper "localized" graph) in comparison to the ordinary diffusion process (lower graph) embedded in one dimension $(D=1, f=1$ and $\left.A(0)=\frac{1}{2}\right)$.

\section{Conclusions}

In our extended facilitated kinetic Ising model we find a (quasi-)localization due to the coupling of the diffusive dynamics for data packages with the existence of the active cells at sufficiently low temperatures. Because their 
part of all cells is small in this case so that the diffusion almost stops. There-

fore, the packages are fixed at or near the initial position, information cannot be widespread. Thus, we may separate two well-distinguished time regimes. First of all the fast flip (or active-passive) relaxation takes place. The higher the restriction number $f$ the more the relaxation time slows down (compare Eq.(18)). Then after a while of the relaxed system of active-passive states sticking the packages the influence of the (slow) diffusion is remarkable, equilibrates the packages concentration (information) of the total system. However, for high enough temperature there are a sufficient part of active cells so that the coupled diffusion can fasten the equilibration and information can easily spread through the network. Like in [24 for the Fredrickson-Andersen model shown mean-field approximation may provide false results. The meanfield solution leads to a dynamics which completely breaks down below a critical (interconnectivity) temperature. That means below a critical (interconnectivity) temperature information would spread through a fixed network like through a sponge (Bond percolation results would apply to this case, see e.g. [25].). Here, we exploit a more sophisticate approximation, taking local processes into account. But we expect that the found quasi-localization alters in a permanent localization at low temperature if $f>z$. Because thus it exist stable clusters of passive cells at any temperature in contrast to the case $f \leq z$ where all cells can be activated [24]. To prove this result one may study the theorem of van Enter [26] describing a bootstrap percolation model 27] (or diffusion percolation 28]).

\section{References}

[1] C.Godrèche, Solids far from Equilibrium (Cambridge University Press, Cambridge, 1992)

[2] V.Privman, Nonequilibrium Statistical Mechanics in One Dimension (Cambridge University Press, Cambridge, 1997)

[3] D.Matthis and M.L.Glasser, Rev. Mod. Phys. 70, 979 (1998)

[4] G.M.Schütz, Exactly Solvable Models for Many-Body Systems far from Equilibrium, Phase Transitions and Critical Phenomena, Eds. C.Domb and J.Lebowitz (Academic Press, London, 2000) 
[5] M.Schulz and S.Trimper, J. Stat. Phys. 94, 173 (1999)

[6] C.Pigorsch, M.Schulz and S.Trimper, Int. Journal of Mod. Physics B 13, 1379 (1999)

[7] G.H.Fredrickson and H.C.Andersen, Phys. Rev. Lett. 53, 1244 (1984)

[8] G.H.Fredrickson and H.C.Andersen, J. Chem. Phys. 84, 5822 (1985)

[9] G.H.Fredrickson, Ann. Rev. Phys. Chem. 39, 149 (1988)

[10] S.Butler and P.Harrowell, J. Chem. Phys. 95, 4454 (1991)

[11] M.Schulz and P.Reinecker, Phys. Rev. B 48, 9369 (1993)

[12] A.Heuer, U.Tracht and H.U.Spiess, J. Chem. Phys. 107, 3831 (1997)

[13] D.Stauffer, Int. Journal of Mod. Physics C 11, 1081 (2000)

[14] S.Lawrence and C.L.Giles, Nature 400, 107 (1999)

[15] A.Kirman, J. Evol. Econ 4, 339 (1997)

[16] S.Redner, Euro. Phys. Journal B 4, 131 (1998)

[17] M.Schulz and S.Trimper, Phys. Rev. E 57, 6398 (1998)

[18] M.Schulz, P.R.S.Sharma and H.L.Frisch, Phys. Rev. B 52, 7195 (1995)

[19] M.Doi, J. Phys. A 9, 1465 (1976)

[20] M.Doi, J. Phys. A 9, 1479 (1976)

[21] S.Sandow and S.Trimper, Eurosphys. Lett. 21, 799 (1993)

[22] G.Schütz and S.Sandow, Phys. Rev. E 49, 2726 (1994)

[23] M.Schulz and S.Trimper, J.Phys. A: Math. Gen. 29, 6543 (1996)

[24] C.Pigorsch, J.C.Kimball and H.L.Frisch, Phys. Rev. B 59, 3196 (1999)

[25] D.Stauffer and A.Aharony, Introduction to Percolation Theory, 2nd Edition (Taylor \& Francis, London, 1994) 
[26] A.C.D.van Enter, J. Stat. Phys. 48, 943 (1988).

[27] J.Adler and A.Aharony, J. Phys. A 21, 1387 (1988)

[28] J.Adler, Physica A 171, 453 (1991) 